\title{
Yielding and fracture mechanisms of nanowires
}

\author{
H. Mehrez and S. Ciraci \\ Department of Physics, Bilkent University, Bilkent 06533, Ankara, Turkey
}

(Received 17 July 1997)

\begin{abstract}
This paper presents a detailed analysis of atomic structure and force variations in metal nanowires under tensile strain. Our work is based on state of the art molecular dynamics simulations and $a b$ initio self-consistent field calculations within the local density approximation, and predicts structural transformations. It is found that yielding and fracture mechanisms depend on the size, atomic arrangement, and temperature. The elongation under uniaxial stress is realized by consecutive quasielastic and yielding stages; the neck develops by the migration of atoms, but mainly by the sequential implementation of a new layer with a smaller cross section at certain ranges of uniaxial strain. This causes an abrupt decrease of the tensile force. Owing to the excessive strain at the neck, the original structure and atomic registry are modified; atoms show a tendency to rearrange in closed-packed structures. In certain circumstances, a bundle of atomic chains or a single atomic chain forms as a result of transition from the hollow site to the top site registry shortly before the break. The wire is represented by a linear combination of atomic pseudopotentials and the current is calculated to investigate the correlation between conductance variations and atomic rearrangements of the wire during the stretch. The origin of the observed "giant" yield strength is explained by using results of the present simulations and $a b$ initio calculations of the total energy and Young's modulus for an infinite atomic chain.
\end{abstract} [S0163-1829(97)01843-2]

\section{INTRODUCTION}

The properties of condensed systems with nanometer dimensions can be rather different from those of the bulk. The effects of reduced size and dimensionality, and properties emerged therefrom have been investigated actively in the past decade. ${ }^{1}$ Connective necks or atomic size wires between sample and metal tip displaying reduced dimensionality and having dimension in the range of Fermi wavelength, $\lambda_{F}$, have been produced in scanning tunneling microscopy (STM) and atomic force microscopy (AFM) operating under extreme tip-sample interaction ${ }^{1-7}$ and also by mechanical break junction. ${ }^{8}$ Because of the unusual transport and mechanical properties, these wires have been subject of several studies. $^{2-16}$ The two-terminal electronic conductance, $G$ showed a staircaselike variation with the stretch, and the yield strength, $\sigma_{Y}$ measured shortly before the break has been found much higher than that of the bulk. The sudden jumps of conductance in the course of stretch have been taken as the realization of quantized conductance at room temperature and thus have created much popularity. However, recent experiments ${ }^{15,16}$ have provided evidence that the observed sudden falls of conductance and tensile force are correlated. Much earlier, Ciraci and Tekman, ${ }^{11,12}$ predicted that sudden changes of $G$ originate from the discontinuous variation of the contact area.

Since a one-dimensional (1D) electron system has the density of states, $D(\epsilon) \sim \epsilon^{-1 / 2}$, the density of states of a perfect metal nanowire can be expressed as $D(\epsilon) \sim \sum_{i} \Theta\left(\epsilon-\epsilon_{i}\right)\left(\epsilon-\epsilon_{i}\right)^{-1 / 2}$; it has peaks when $\epsilon=\epsilon_{i}$. Furthermore, the level spacing, $\Delta \epsilon=\epsilon_{i+1}-\epsilon_{i}$, becomes strongly dependent on the size and the geometry of the nanostructure. Such an effect was investigated earlier by Ciraci and Batra, ${ }^{17}$ who found that surface energy, work function and bandwidth show discrete changes upon the change of size. In this respect, one can argue that even the changes of the diameter of the connective neck are closely correlated with the occupancy of transversally confined electronic states. The narrowest diameter of the nanowire prior to the break is only few angstroms; it has the length scale $\lambda_{F}$, where discontinuous (discrete) nature of the metal dominates over its continuum description. Since $\Delta \epsilon$ is in the range of $\sim 1 \mathrm{eV}$ in this length scale, the peaks of $D(\epsilon)$ of the connective neck becomes stronger and hence the transversal quantization of states become easily resolved even at room temperature. Furthermore, any change in the atomic structure or in the diameter induce significant changes in the level spacing, that in turn, may lead to detectable changes in the related properties. For example, the ballistic electron transport through nanowire is closely related with its atomic structure and diameter at the narrowest part of the connective neck. It becomes now clear that the mechanical properties, in particular, the yielding mechanisms of the nanowires are quite different from those of the bulk material.

The atomic scale simulations of the metallic nanocontacts were carried out first by Sutton and Pethica ${ }^{3}$ and by Landman and his co-workers. ${ }^{4}$ Because of recent transport experiments ${ }^{5-8}$ which posed new questions, the atomic structure and mechanical properties of nanowires produced by pulling the STM tip from an indentation have become the subject of renewed interest. Various electronic and atomic processes that are resulted from elastic and plastic deformation of nanowire are not fully understood yet, and deserve further study. It is now well accepted that the understanding of interesting features in the conductance variations as well as unusual mechanical properties require the precise knowledge of atomic structure in the course of stretch. ${ }^{18}$ It is also most interesting to know how a nanostructure behaves under high tensile stress, in what direction the atomic structure 


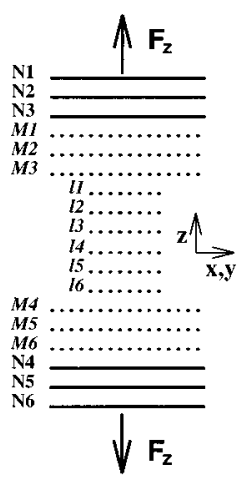

\begin{tabular}{|l|ll|ll|ll|l|}
\hline SQ & $\mathrm{N} 1$ & $\mathrm{~N} 2$ & $M 1$ & $M 2$ & $l 1$ & $l 2$ & Layer \\
& 145 & 145 & 144 & 144 & 13 & 12 & $\mathrm{Cu}(100)$ \\
& 144 & 145 & 145 & 145 & 13 & & \\
\hline \multirow{3}{*}{$\mathrm{N} 3$} & $\mathrm{~N} 4$ & $M 3$ & $M 4$ & $l 3$ & $l 4$ & Structure \\
& 145 & $\mathrm{~N} 6$ & $M 5$ & $M 6$ & $l 5$ & $l 6$ & \\
\hline & 143 & 143 & 143 & 143 & 12 & 12 & \\
& 143 & 143 & 143 & 143 & 13 & 12 & $\mathrm{Cu}(111)$ \\
& 143 & 143 & 143 & 143 & 12 & 13 & \\
\hline
\end{tabular}

FIG. 1. Schematic description of the $\mathrm{Cu}$ nanowires. The layers labeled by $N, M$, and $l$ are described in the text. The number of atoms in each layer and their ideal 2D structures before pulling are indicated. The $x$ and $y$ axes lie in the atomic $[(001)$ or (111)] planes. The $z$ axis coincides with the axis of the wire; the tensile force $F_{z}$ is applied along the $z$ axis.

evolves in the absence of constraint of translational periodicity, and how the mechanical properties, in particular, the yield strength and elastic constants of a nanowire are correlated with its atomic and electronic structure.

In this work, the yielding and fracture mechanisms of nanowires which are pulled by an external agent have been investigated. To understand the origin of these mechanisms and abrupt force variations, ${ }^{15,16}$ an extensive analysis of atomic structure have been presented by performing atomic simulations of pulling. In particular, the motion of the neck atoms, their coordination numbers and also the structure of atomic layers have been examined during the abrupt force variations. The effect of the initial atomic structure and temperature have been investigated. We carried out the state of the art molecular dynamics (MD) calculations for the $\mathrm{Cu}$ nanowires made from (001) and (111) atomic planes. Since the connective neck having few atoms at the narrowest part of its cross section is a subject of interest, we dealt with wires that have small cross sections. To reveal the effect of size on the mechanical properties, in particular, to understand physical phenomena underlying the "giant" Young's modulus observed in thin wires shortly before the break, we carried out $a b$ initio calculations by using self-consistent field (SCF) pseudopotential method within local density approximation. To analyze the correlation between the conductance and the atomic structure, we first constructed a realistic potential corresponding to the atomic structure obtained from the MD simulations and calculated the conductance as a function of stretch by using transfer matrix method.

\section{METHOD FOR MD SIMULATIONS}

The wires we studied have two ends which are connected by a neck. The description of their structures are summarized in Fig. 1. Last three layers at both ends $\left(N_{1}, N_{2}, N_{3}\right.$ and $N_{4}, N_{5}, N_{6}$ ) are rigid; all the atoms in these layers are kept fixed during the MD-relaxation steps. These fixed three layers at both ends are assumed to be connected to the external agent (or grips) which applies the tensile stress. Atoms in the following three layers adjacent to the fixed ones $\left(M_{1}, M_{2}, M_{3}\right.$ and $\left.M_{4}, M_{5}, M_{6}\right)$ and those of the neck $(l 1, l 2, l 3 \ldots)$ are identified as dynamic atoms and are fully relaxed during the MD steps. We considered two different crystal structures. The first nanowire indicated by $S Q$ is initially formed from $\mathrm{Cu}(001)$ atomic layers and investigated at $T=300 \mathrm{~K}$. The wire itself is represented by a periodically repeating system in the $(x y)$ plane. The $z$ axis is taken to be parallel to the axis of the nanowire. The second nanowire
$H X$, which is made from $\mathrm{Cu}(111)$ atomic planes, is investigated at $T=300 \mathrm{~K}$ and $T=150 \mathrm{~K}$. In order to avoid effects of corners with low coordination number, the atomic layers are taken quasicircular. The interatomic interactions are treated by the embedded atom (EA) model potential. ${ }^{19-21}$

The pulling off (or stretch) is realized by displacing the top fixed layers $\left(N_{1}, N_{2}, N_{3}\right)$ solidly along the the $z$ direction in increments of $\Delta z$ while keeping the other end fixed. We believe that such a process of pulling off is similar to the real stretching phenomena and hence provides realistic simulations. Whereas different schemes of stretching have been also used in previous works. ${ }^{3}$ Between two consecutive increments (stretches) of $\Delta z$, the dynamic atoms of the wire (i.e., those of $M^{\prime} s$ and $l^{\prime} s$ ) are relaxed to find their next "steady-state" condition. The "steady-state" condition is identified by the fact that the system is allowed to evolve dynamically until no significant variation in physical quantities (temperature, force, etc.) are observed beyond natural fluctuations. The relaxation process is realized in $k_{r}$ steps, each lasting $\Delta t$ seconds. In every time step, Newton's equation is solved to find new position of atoms while temperature is controlled using Hoover drag. The lower limit of $\Delta t$ is determined from the mobility of atoms in the solid. In the atomistic simulations $\Delta z, \Delta t$ and temperature $T$ are crucial parameters. While the STM experiments have been carried out with the characteristic strain rate or tip retraction velocity in the range of $10^{-10} \mathrm{~m} / \mathrm{sec}$, the velocity of the stretch $v_{s}=\Delta z /\left(k_{r} \Delta t\right)$ used in the MD simulations ranges from $0.4-60 \mathrm{~m} / \mathrm{sec}$. This dramatic difference between experiment and MD simulations has been questioned by Sutton. ${ }^{22}$ In spite of this dramatic difference it appears that the simulations carried out with relatively higher speed can reveal the main features of the atomic rearrangements. The increment, $\Delta z$ by itself is important; the break of the junction can be favored by relatively larger $\Delta z$. Continuous and homogeneous stretching of the junction by using very small $\Delta z$ followed by few relaxation steps have also been used. Even though the experimental velocities can not be achieved, we nevertheless examined how the yielding and fracture mechanism depend on the velocities by carrying out the MD simulations with $\Delta t=3 \times 10^{-15}, 1.5 \times 10^{-14}$, and $10^{-13} \mathrm{sec}$ corresponding to $v_{s}=0.05,0.01$, and $0.004 \mathrm{~m} / \mathrm{sec}$, respectively. ${ }^{23}$ The tensile force $F_{z}$, that corresponds to the force exerted by the pulling agent to the wire (and is the attractive force between the top layers and the rest of the wire) is calculated after averaging over the last 8000 relaxation steps. The overall features of the results, in particular, 

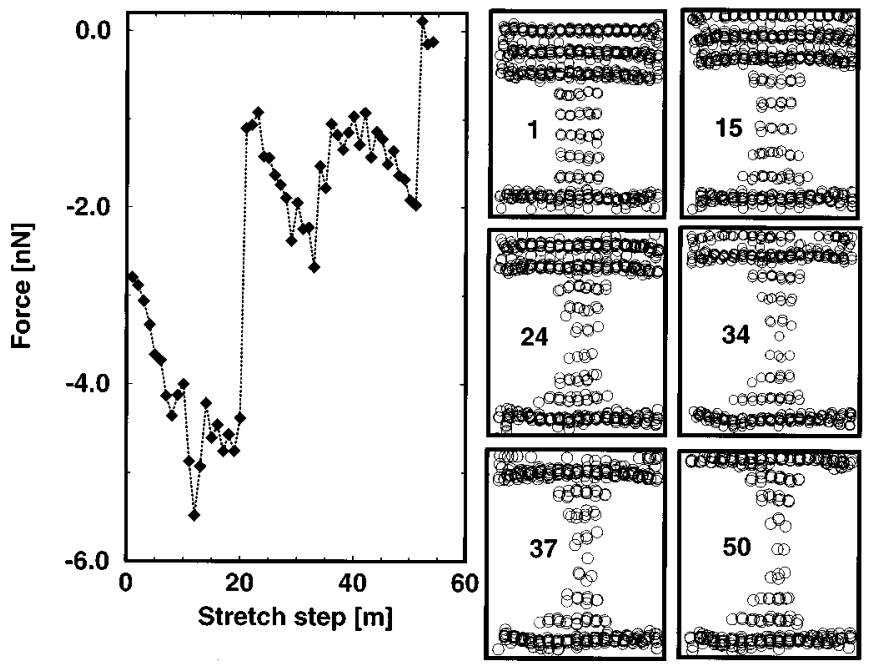

the character of the force variation, yielding mechanisms and atomic rearrangements were essentially the same for different stretching time steps (or velocities). There are some features that depend on $\Delta t$ (or $v_{s}$ ) which will be discussed in the next section.

\section{RESULTS OF MD SIMULATIONS}

We examine first the elongation and various physical events during the stretch of the nanowire, $S Q$ at $T=300 \mathrm{~K}$ (see Fig. 1). The interlayer distance between the bulk $\mathrm{Cu}(001)$ layers is $c \sim 1.8 \AA$ (half of the cubic lattice parameter, i.e., $\sim a / 2)$. Initially, the layers of the wire have $A-B-A-$ $B$ stacking sequence; the atoms of the $B$ layer face the hollow sites (the center of the square unit cells) of the adjacent A layers. We specify this stacking as the H-site registry. The increments of length at each stretch step is taken to be $0.1 \AA$ $(\Delta z=0.1 \AA$ ). We first present the results corresponding to $v_{s}=0.004 \mathrm{~m} / \mathrm{sec}$ (or $\Delta t=10^{-13} \mathrm{sec}$ ) but contrast them with those obtained with larger $v_{s}$. In Fig. 2, we illustrate the variation of $F_{z}$ as a function of stretch $m \Delta z$ ( $m$ being an integer number), where the negative sign is taken for the tensile force. The side views of atomic structure are also shown in the same figure. During the initial stage of stretch, the tensile force increases with increasing $m$ between two consecutive jumps of $F_{z}(m)$. This is the quasielastic stage. ${ }^{24}$ At the end of each quasielastic stage involving approximately 17-20 increments (that corresponds approximately to c) the atomic structure in the neck becomes disordered and concomitantly the tensile force decreases abruptly. This structural instability which occurs at high strain level, is the yielding stage. The yielding stage and disordered atomic structure of the neck lasts until a new layer with relatively smaller cross section is generated. The formation of the neck layers at fairly regular intervals was predicted earlier. ${ }^{6}$ The overall behavior of $F_{z}(m)$ is in agreement with the experimental results. ${ }^{4,15,16}$ We now examine these in detail.

At the last increment before the first jump in $F_{z}(m)$ $(m \sim 19)$, the layer structure in the neck is destroyed, and becomes disordered; but it is recovered after a few increments, at $m \sim 24$ with the creation of a new layer. ${ }^{25}$ At the end of this transformation, the cross section of the neck is reduced from eight atoms to five atoms; whereas the cross

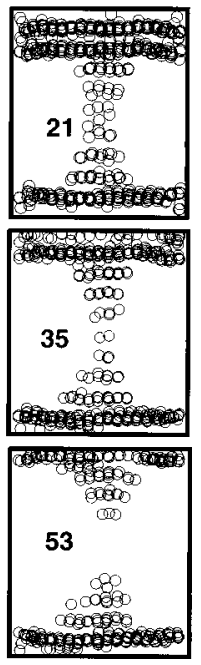

FIG. 2. The variation of tensile force $F_{z}$ (in nanoNewtons) with the strain or elongation along the $z$ axis applied by pulling $N_{1}, N_{2}, N_{3}$ end layers of the $\mathrm{Cu}(001)$ nanowire $(S Q)$ by $m \Delta z$. The side views show the atomic positions at relevant stretch values $m$. The MD simulations are performed at $T=300 \mathrm{~K}$ by using $\Delta t=10^{-13} \mathrm{sec}$. section of neck layers adjacent to end layers ( $l 1$ and $l 5)$ is not altered. We note that during the initial stage of stretch, $1<m<19$, the cross section of the neck is reduced by the migration of atoms towards the ends of the wire. Not only the tensile force, but also the tendency to reduce the surface energy is the driving force for the atomic migration. The local reduction of the cross section due to stretching causes the average of $\left|F_{z}\right|$ to reduce, and hence the interlayer spacings between $M_{3}$ and $l 1$ (and similar spacing at the other end) to decrease. The layer structure of the neck is maintained until $m \simeq 33$. Beyond that point each increment of stretch by $\Delta z$ causes one atom from the central layer to migrate and stay in the adjacent interlayer spacing which already became wide open due to pulling from $m=25$ to $m=33$. This way a new "layer'" with two atoms is formed. As a result, the cross section of the connective neck is further reduced with three central layers formed by 5,2,3 atoms, respectively. Owing to the repulsive force induced between the new layer and adjacent ones, $\left|F_{z}\right|$ decreases abruptly. The asymmetric distribution of atoms in the neck layers may be due to a dynamical effect and due to stretch of the wire from one end, as well. However, in the steps from $m=35$ to $m=38$, the asymmetry is lifted by a peculiar, transient event: One of the two atoms in the neck layer created at $m=35$ jumps back to the layer it emerges. During the following increments, the single atom neck is strengthen by the inclusion of one atom from another layer so that the necking becomes uniform by the layers including 4,2,4 atoms. The implication of this event to the measured conductance will be examined in Sec. IV. The two-atom neck becomes stable until the break; but the atoms rotate in the (xy) plane and they become slightly inclined in the $z$ direction. Such a fluctuation in configuration may give rise to changes in the conductance. It is also seen that the narrowest cross section of the neck prior to the break can have two atoms.

Next we examine atomic rearrangements and structural changes in every atomic layer, which become gradually nonplanar under tensile stress. The arrangements of atoms in the layers are shown in Fig. 3. In our study we have found that up to $m=12$, the $\mathrm{Cu}(001)$ lattice structure in the neck layers exhibits small deformations. The original stacking and registry are continued despite some deformation. Beyond a cer- 


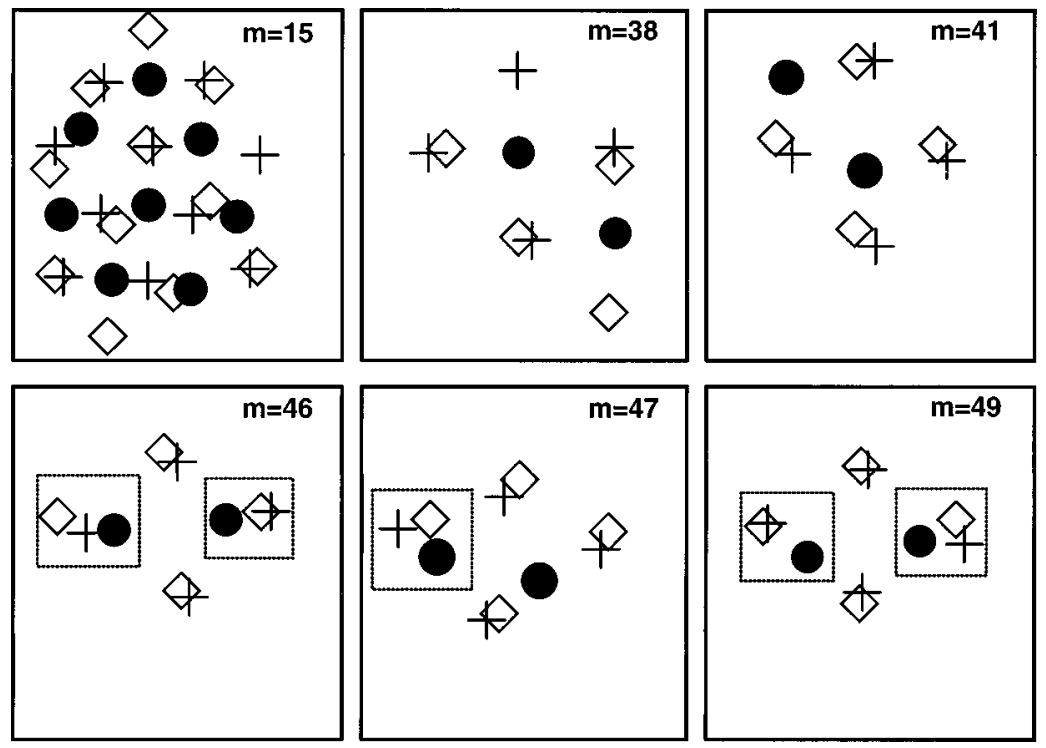

FIG. 3. The top views of three layers at the neck showing atomic positions and their relative registry at different levels of stretch. $m=15 \mathrm{oc}$ curs before the first yielding stage. The atomic positions in the layers $l 2, l 3$, and $l 4$ are indicated by,$+ \boldsymbol{\bullet}$, and $\diamond$, respectively. $m=38$ and $m=41$ occur after the second yielding stage. $m=46,47$ and $m=49$ show the formation of bundle structure. In the panels $m=38-49$ the positions of atoms in the third, fourth and fifth layers are indicated by,$+ \boldsymbol{O}$, and $\diamond$, respectively. Atomic chain in a bundle are highlighted by dotted boxes.

tain stretch, the neck atoms evolve to a structure that deviates from the usual $A-B-A-B$ stacking sequence of the ideal $\mathrm{Cu}(001)$ planes. The $2 \mathrm{D}$ square lattice changes and then looks like a hexagonal one. Upon further elongation of the nanowire, the rest of the neck undergoes the same deformation. The tendency towards a 2D hexagonal-like lattice is mediated by the reduced interlayer interaction as a result of stretch; the atoms tend to take relatively lower energy configuration with higher coordination number in the quasiindependent layer behavior. The original $A-B-A-B$ sequence of the $\mathrm{Cu}(001)$ planes at the neck can be recovered only after the second additional layer is formed.

An interesting situation owing to the reduced interlayer interaction and excessive tensile strain appears in the last stage of stretch: The $H$-site registry disappears eventually shortly before the break and hence the atoms of the adjacent neck layers tend to face the top (T) sites. This way the atoms in the neck are aligned to form atomic chains as shown in Fig. 3. As a result, the crystal-like structure and original registry at the neck are replaced by a bundle of atomic chains. In some circumstances, the single atomic chain can form instead of a bundle. In the present case, the transition from the $H$ site to the $T$ site leading to the bundle structure occurs under high strain, whereas, the energy difference is only $\sim 0.2 \mathrm{eV}$. Earlier, ab initio calculations also confirmed that the $T$-site registry is only a local minimum occurring at relatively larger interlayer separation for the $\mathrm{Al}(111)$ and $\mathrm{Al}(001)$ slabs. ${ }^{26}$ However, in other materials oriented in certain atomic planes, the break can take place before such a transition. The transition from the $H$-site registry to the $T$-site registry leading to a bundle of chain structure is interesting not only for the point of view of structural modification, but also for different properties (such as "giant'" yield strength $^{15,16}$ and positive slope of conductance variations ${ }^{8,27}$ ). These effects will be examined in Sec. IV and Sec. V.

It is now in order to compare the results with those obtained by $\Delta t=3 \times 10^{-15} \mathrm{sec}\left(v_{s}=0.05 \mathrm{~m} / \mathrm{sec}\right)$. The evolution of the atomic structure and the $F_{z}(m)$ curves with jumps for $m=19,35,52-55$ display similar features. However, in the latter case the wire can be stretched relatively longer until the break, and $F_{z}(m)$ curve exhibits higher fluctuations. For ex- ample, while the first three quasielastic and yielding stages are essentially the same, the single atom neck in the case of $\Delta t=3 \times 10^{-15}$ survives until $m=75$.

The wire that was made initially from the $\mathrm{Cu}(111)$ and specified by $H X$ in Fig. 1 has overall features of the yielding mechanism at $T=300 \mathrm{~K}$, which are similar to the above $\mathrm{Cu}(001)$ orientation. In Fig. 4, we show the force variation with stretch, and the side views of the nanowire at the moment of structural transformations. As in the previous case, the force curve depicts consecutive quasielastic and yielding stages. Initially, the quasielastic stages before the first and second structural transformation are rather linear with strain. This is not so in the third stage before the break, in which it is not possible to identify a stable layer structure throughout. In the last stage, the atoms in the narrowest part of the neck move between layers and the structure becomes generally disordered, but the layer structure is recovered only temporarily in the course of stretch. As a result, $F_{z}(m)$ is linear only in short intervals, but exhibits significant jumps. In this last stage of stretch, the strain is almost accommodated by the central part of the neck. We see that before the break, the neck transforms into an atomic chain. The 2D hexagonal-like structure $^{28}$ is quite robust for the layers adjacent or close to the end layers $M_{3}$ and $M_{4}$. Here the $A-B-C$ stacking sequence and the $H$-site registry are forced by these end layers. In the central part of the neck, the distortion of the $2 \mathrm{D}$ hexagonal lattice structure becomes severe and the $A-B-C$ sequence disappears, but the $H$-site registry is kept after the yielding stage. However, the atomic chains form as a result of the transition to the $T$-site registry in the last stage of stretch. As for the simulations with $\Delta t=3 \times 10^{-15} \mathrm{sec}$ $\left(v_{s}=0.05 \mathrm{~m} / \mathrm{sec}\right)$, we obtained similar features in $F_{z}(m)$ and in the evolution of the atomic structure, except that the wire was stretched relatively $(0.7 \AA)$ longer until the break.

When the above $\mathrm{Cu}(111)$ wire (labeled by $H X$ in Fig. 1) is stretched at $T=150 \mathrm{~K}$ exhibits features, some of which are dramatically different from those at room temperature. For example, the same wire undergoes approximately five yielding stages resulting in an elongation of $9.5 \AA$; whereas at room temperature, it had only three yielding stages with 6 $\AA$ elongation. Apparently, while the necking is faster and 

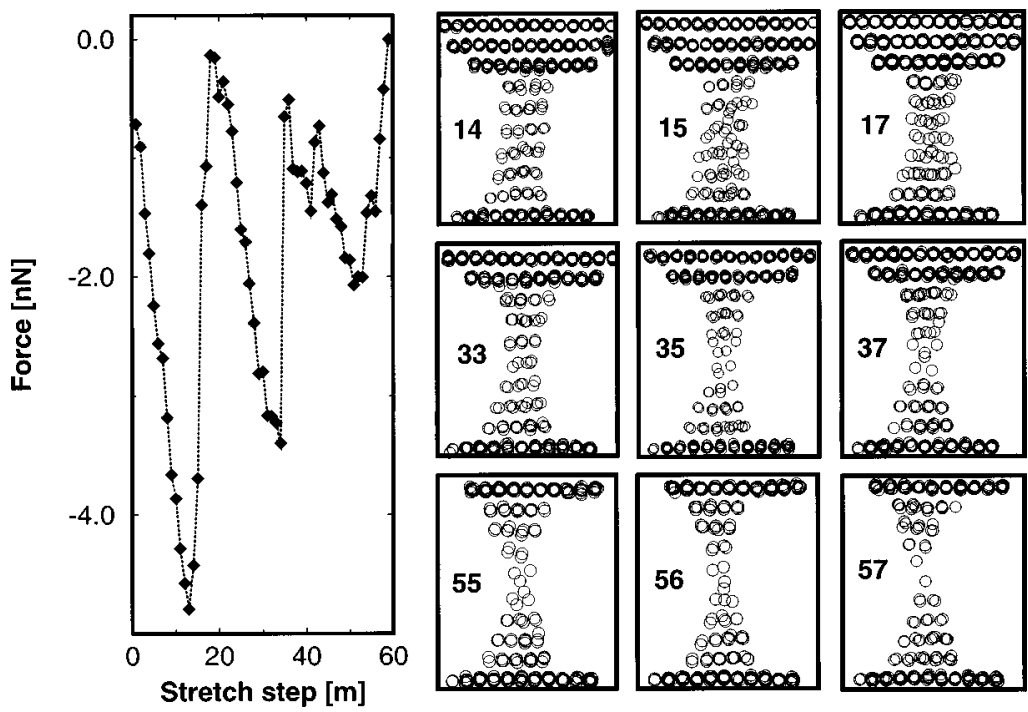

FIG. 4. The variation of tensile force $F_{z}$ with the stretch and side views showing the atomic positions at relevant stretch values $m$, for the $\mathrm{Cu}(111)$ nanowire $(H X)$. The MD simulations are performed at $T=300 \mathrm{~K}$ with $\Delta t=10^{-13} \mathrm{sec}$. sharper and hence is more trumpetlike at room temperature, the neck is longer at low temperature. We explain this situation by faster atomic motion towards the ends of the wire at room temperature. Another feature which does not exist at room temperature is a new type of structural transformation. The $F_{z}(m)$ curve and some relevant side views of the wire shown in Fig. 5 distinguish different types of yielding mechanisms. The first and second yielding stages are similar to the previous cases in which the structure undergoes disorder-order transformations during the generation of a new layer. At $m=15$ the well defined layer structure becomes broader and slightly disordered, and a new layer forms at $m=16$. This yielding stage occurs more abruptly with least necking; the size of the layers decreases by 2-3 atoms. A similar process repeats at $m=35$, and creates a new layer at $m=36$. Again, in this process most of the layers are affected and reduced by two atoms with no local significant necking. The hexagonal rings and $A-B-C$ stacking sequence of the $\mathrm{Cu}(111)$ planes are robust near the end layers $\left(M_{3}\right.$ and $M_{4}$ ). At the central layers hexagonal rings persist, ${ }^{28}$ but the $A-B-C$ stacking sequence and its characteristic registry between adjacent layers are destroyed. The shape of the layers in the ( $x y)$ plane is distorted. For $m>40$, an intermediate situation occurs, in which not only elastic stretching but also new mechanisms contribute to the local elongation of the wire: Hexagonal rings change into pentagonal rings; the latter ring becomes staggered to the rings in the adjacent layers. Moreover, one layer at a time (starting from the central layer) ejects one atom to the interlayer space. This way a column (or chain) of atoms is formed along the axis of the neck. The projection of this chain in the $(x y)$ plane is almost a single point near the centers of pentagonal rings as shown in Fig. 6(a) and Fig. 6(b). Out of five yielding stages, the last three exhibit these features. Because different mechanisms are involved in the stretching, the linear variation of $F_{z}(m)$ in the quasielastic stage is not as distinguishable as those occurring at the beginning of stretch. At prolonged stretch $(m>75)$, the number of atoms in the neck layers are reduced and the layer structure becomes more distorted; staggered pentagonal rings change into triangular rings again with staggered orientation [see Fig. 6(c)]. A few steps before break $(84<m<94)$, a single atomic chain forms eventually. We understand that at low temperature, the mobility of atoms are not suitable for massive changes, so the neck can accommodate the applied strain by these new intermediate structures which require least atomic rearrangement. The MD simulations of the same wire at $T=150 \mathrm{~K}$ and with $\Delta t=3 \times 10^{-15}$ sec $\left(v_{s}=0.05 \mathrm{~m} / \mathrm{sec}\right)$ yield the $F_{z}(m)$ curve that displays

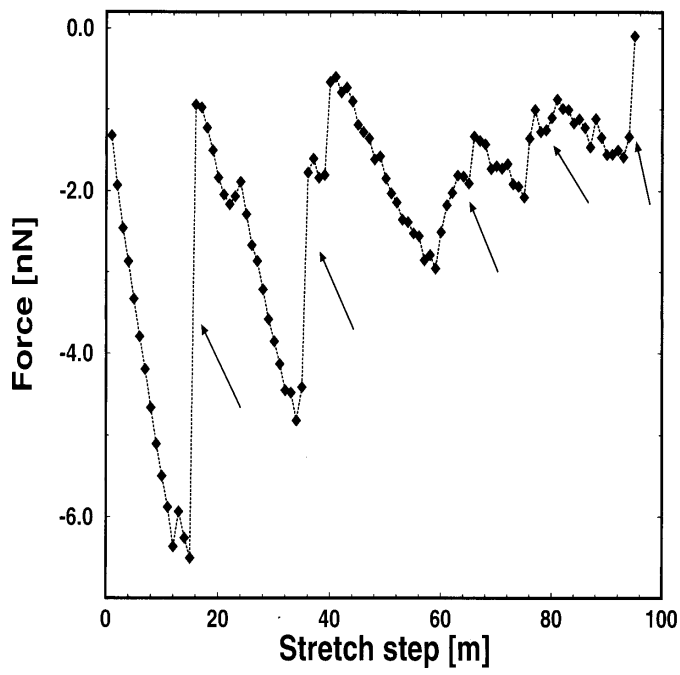

FIG. 5. Variation of tensile force $F_{z}$ and side views showing atomic positions at relevant stretch steps $m$, for the $\mathrm{Cu}(111)$ nanowire $(H X)$. The MD simulations are performed at $T=150 \mathrm{~K}$ with $\Delta t=10^{-13}$ sec. 



FIG. 6. Top views of atoms in the three central layers (indicated by $+, \bigcirc, \diamond$ ) of the neck show atomic positions and their relative registry for the $\mathrm{Cu}(111)$ nanowire $(H X)$ stretched at $T=150 \mathrm{~K}$. Panels (a), (b), and (c) correspond to $m=43,48$, and 81, respectively, and $\Delta t=10^{-13}$ sec. Panels (d),(e), and (f) are for $m=43,48$, and 85 , respectively, and $\Delta t=3 \times 10^{-15} \mathrm{sec}$. overall features similar to those explained above. This curve and some relevant side views of atomic structure are shown in Fig. 7. The first three yielding stages occur approximately at the same level of stretch $m$. Beyond $m>60$, the elastic and yielding stages are not clearly distinguishable. As seen in Fig. 6(d) and Fig. 6(e), the staggered hexagonal structure with atomic chain passing through the center occur in $40 \leqslant m \leqslant 80$. However, the hexagonal rings cannot transform to pentagonal ones as in the previous case (with $\Delta t=10^{-13}$ $\mathrm{sec}$ ) since the atoms at the center are displaced slightly off layer, but cannot overcome the barrier to reach the stable equilibrium position near half way between adjacent layers. In $80<m<90$, atoms are arranged on staggered triangle leaving a hole at the center as seen in Fig. 6(f). Eventually, this structure changes into a single atomic chain at the neck just before the break.

\section{ELECTRON TRANSPORT IN NANOWIRES}

So far the present calculations demonstrated that in the course of stretch $F_{z}(m)$ [or $\left.F_{z}(s)\right]$ drops suddenly owing to the yield leading to the formation of a new layer with relatively smaller cross section or due to other type of atomic rearrangements. Here we will investigate the effect of these structural transformations on the two-terminal conductance. As far as the electronic transport is concerned, a metal nanowires can be viewed as a constriction between two electrodes or reservoirs. In this constriction, the electronic potential $V(r)<E_{F}$, and the energies of the electrons have quantized values $\epsilon_{i}$. Here one can give a simple understanding of the current transport between reservoirs in terms of an "ideal situation" if $V(r)$ is uniform and hence yields unique energy quantization throughout the constriction, or in the adiabatic limit $\epsilon_{i}=\epsilon_{i}(z)$. Under these circumstances, each current transporting state between the reservoirs with $n$ fold degeneracy below $E_{F}$ can be viewed as an $n$ fold conduction channel with an ideal conductance of $n\left(2 e^{2} / h\right)$. Shortly before the break, the radius of the connective neck is in the range of $\lambda_{F}$, and its cross section comprises only few (1-3) atoms. Under these circumtances, the current transporting states have $\Delta \epsilon \sim 1 \mathrm{eV}$ that would easily be resolved at room temperature. The atomic structure revealed in the previous sec-

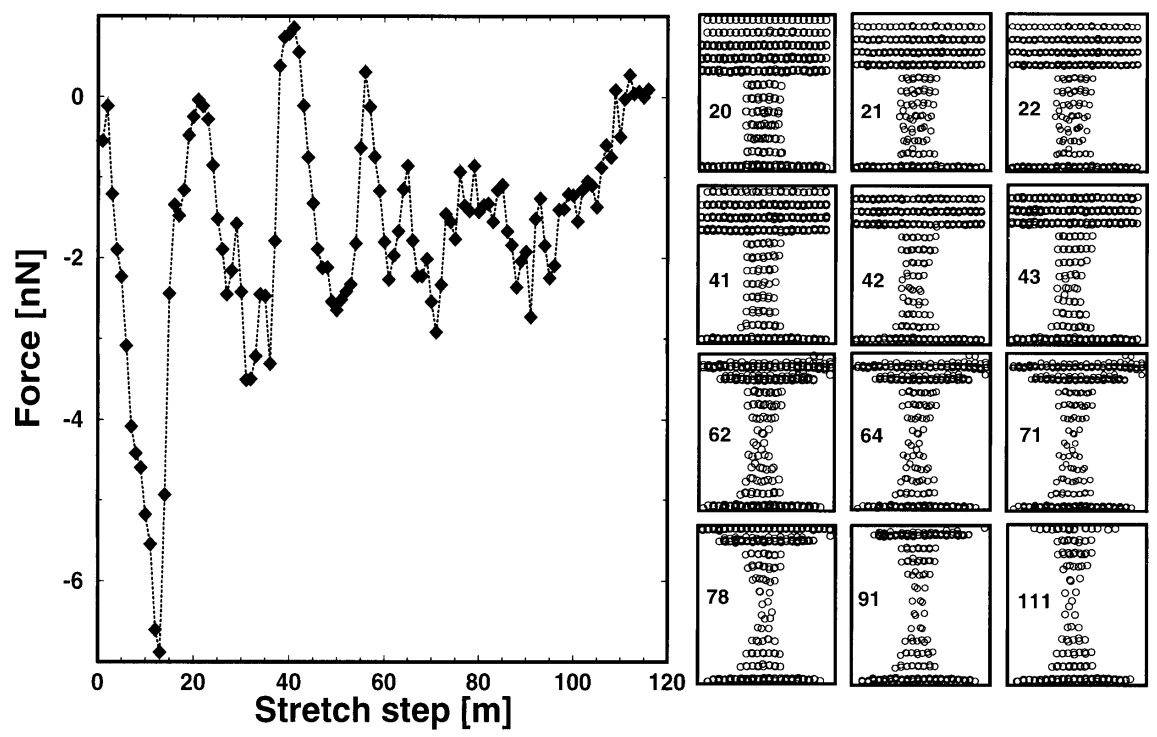

FIG. 7. Same as Fig. 5 except that $\Delta t=3 \times 10^{-15} \mathrm{sec}$. 
tion, as well as the experimental data on the variation of conductance with $s$ indicate that the above ideal conditions cannot be realized in the connective neck even if their diameters can be reduced down to the range of $\lambda_{F}$ to yield wide level spacing. First of all, the length of the uniform region at the narrowest part is smaller than $\lambda_{F}$. This increases the contribution of tunneling and hence smoothes out the sharp step structure that normally occurs when a conduction channel is opened (or closed). An extreme case is, of course, the quantum Sharvin's conductance, ${ }^{12,29,30}$ where the step structure is almost smeared out and plateaus disappeared since the length of the constriction vanishes. Furthermore, $V(r)$ in the wire is nonuniform and strongly roughened due to the irregular atomic arrangement as illustrated in Figs. 2 and 3. Under these circumstances, the channels mix significantly, ${ }^{12}$ the opening of the channels are delayed and $G$ attains values lower than the integer multiples of quantum conductance. ${ }^{12,30,31}$ Then the resulting $G(s)$ curve would trace smoothed structure if the cross section $S$ of the neck were changed continuously with $s$. Apparently, this behavior also is not supported by the $G(s)$ curve obtained experimentally. 5,8

In view of the earlier work ${ }^{11}$ (based on the first 3D calculations of conductance in a constriction between an STM tip and metal surface), recent extensive studies ${ }^{13,14,32}$ and recent experimental results measuring force and conductance simultaneously, ${ }^{15,16}$ the understanding of the features in the experimental $G(s)$ curve requires the calculations of the conductance using a SCF potential corresponding to the detailed atomic structure of the wire. A rigorous SCF calculation in 3D for a realistic $\mathrm{Cu}$ (transition metal) wire is too tedious, however. We nevertheless treated this problem by generating a realistic potential constructed from the linear combination of atomic potentials according to the atomic structure calculated by the present MD simulations. First we calculated the ionic potential of a free $\mathrm{Cu}$ atom, $V_{p}(r)$ from the generalized norm-conserving pseudopotential and the atomic charge density distribution, $q_{a}(r)$ by using Hamann's program. ${ }^{33} \mathrm{We}$ next Fourier transform the atomic potential in order to take into account the electron-electron screening effect in the neck using Thomas-Fermi approximation. At this stage, we define

$$
V_{p}(K)=\frac{1}{(2 \pi)^{3}} \frac{4 \pi}{K} \int_{0}^{\infty} r V_{p}(r) \sin (K r) d r,
$$

where $K$ is $|\vec{K}|$. In order to calculate the potential of the wire corresponding to a given stretch $s$, its charge density at $\vec{r}$ is calculated from the linear combination of atomic charge density $q_{a}(r)$,

$$
q_{s}(\vec{r})=\sum_{i} q_{a}\left[\vec{r}-\vec{R}_{i}(s)\right],
$$

where $\vec{R}_{i}(s)$ are the atomic positions obtained from our MD simulations performed at a given $s$. The index $i$ runs over all atoms of the wire. The potential at this point is

$$
V_{s}(\vec{r})=V_{\mathrm{xc}}\left(\vec{r}, q_{s}\right)+\sum_{i} \int d^{3} \vec{K} \frac{V_{p}(\vec{K})}{\varepsilon\left(\vec{K}, q_{s}\right)} e^{i \vec{K} \cdot\left[\vec{r}-\vec{R}_{i}(s)\right]}
$$

where $V_{\mathrm{xc}}$ is the Ceperley-Alder ${ }^{34}$ exchange-correlation potential and $\varepsilon\left(\vec{K}, q_{s}\right)$ is the Fourier component of the static dielectric function. To this end the potential is transformed to finite wall cylindrical potential through radial averaging and then least square fit. In order to make computations of transversally confined states feasible, the walls of $V_{s}(\vec{r})$ are taken infinite. The effect of this change, is the upward shift of the energy which is corrected by increasing the radius of the infinite cylindrical wall potential, $\rho_{s}(z)$, at any $z$ so that the eigenenergy of the ground state coincides with that of finite wall potential. Eventually, the potential of the wire reads

$$
V_{s}(\rho, z)= \begin{cases}\phi_{s}(z), & \rho \leqslant \rho_{s}(z) \\ \infty, & \rho \geqslant \rho_{s}(z),\end{cases}
$$

with $\rho=\left(x^{2}+y^{2}\right)^{1 / 2}$. The saddle point potential $\phi_{s}(z)$ is an essential ingredient that is omitted in the free and nearly free electron calculations. ${ }^{7,14}$ This potential can be taken into account in the evaluation based on the empirical tight-binding method ${ }^{13}$ by shifting the orbital energies by $\phi_{s}(z)$. We calculate the conductance of the nanowire (that is specified by the stretch $s$ it undergoes) by using the transfer matrix method: We divide $V_{s}(\rho, z)$ into discrete segments of equal widths, and assume that in each segment $z_{j}<z<z_{j+1}, \phi_{s}(z)$, and $\rho_{s}(z)$ take uniform values obtained by averaging therein. Then the longitidunal wave function $e^{i \gamma_{n_{j}}^{s}(z)}$ and transversal wave function with circular symmetry, $\varphi_{n_{j}}^{s}(\rho ; z)$, are solved for each segment. Here the propagation constant is

$$
\gamma_{n_{j}}^{s}(z)=\left\{\frac{2 m}{\hbar^{2}}\left[E-\phi_{s}(z)-\epsilon_{n_{j}}^{s}(\rho ; z)\right]\right\}^{1 / 2},
$$

where $E$ is the energy of the incident electron and $\epsilon_{n_{j}}^{s}(\rho ; z)$ is the eigenenergy of $\varphi_{n_{j}}^{s}(\rho ; z)$. If $E>\phi_{s}(z)+\epsilon_{n_{j}}^{s}$ all the $\gamma_{n_{j}}^{s}$ are real and propagate in the segment. Imaginary $\gamma_{n_{j}}^{s}$ occurring for $\epsilon_{n_{j}}^{s}>E-\phi_{s}(z)$ yield longitidunal waves that decay in the segment, and contribute to the current through tunneling. We start with an incident plane wave $e^{i \vec{k}_{l} \cdot \vec{r}}$ for $z \leqslant 0$ in the left reservoir, and construct the current transporting state in each segment $z_{j}<z<z_{j+1}$ by the linear combination of the longitudinal and transversal states; the coefficients of these states are determined by the multiple boundary matching. The current is calculated by evaluating the current operator corresponding to an incident plane wave and summing it over the Fermi surface. The conductance is obtained within linear response theory.

Figure 8(a) shows the $G(m)$ curve that was calculated for the stretch of the wire $(S Q)$ simulated at $T=300 \mathrm{~K}$ with $\Delta t=10^{-13} \mathrm{sec}$. We note five plateaulike structures with small averaged slope $|\Delta G / \Delta m|$; the conductance changes abruptly between these regions. These and other features can be understood by comparing the $G(m)$ curve with that of $F_{z}(m)$ in Fig. 2, and also by examining the the evolution of atomic structure with stretch. The first fall of $G(m)$ occurs in $7 \leqslant m \leqslant 10$ due to the migration of atoms from the neck towards the shank. In the region $10<m<18$ the averaged slope, $|\Delta G / \Delta m|$ decreases, but as a result of the yield leading to a new layer with smaller cross section in $20<m<24$, $G(m)$ falls suddenly. The third fall starts at $m=34$, where 


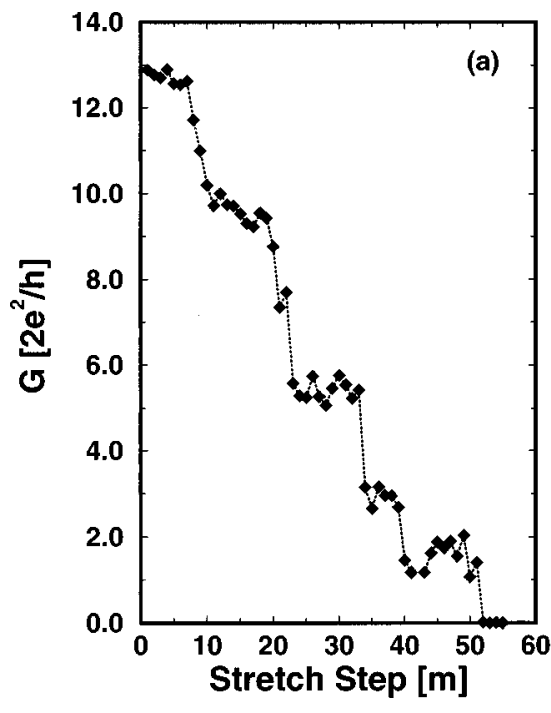

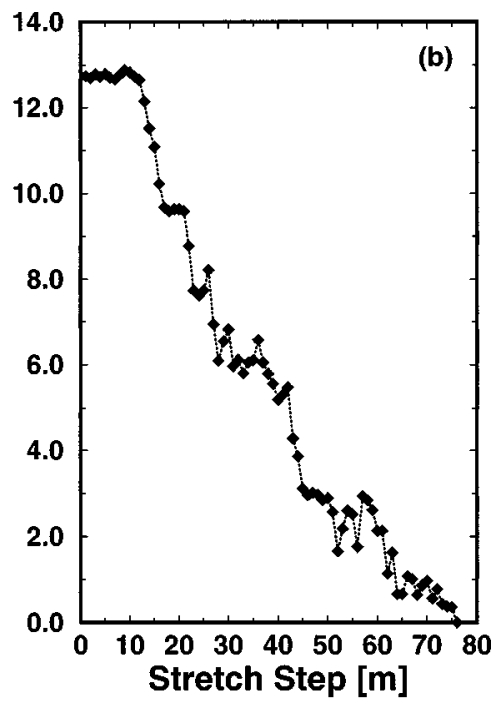

FIG. 8. Variation of the conductance calculated for the $\mathrm{Cu}(001)$ nanowire $(S Q)$ stretched at $T=300$ K. (a) $\Delta t=10^{-13} \mathrm{sec}$ and (b) $\Delta t=3 \times 10^{-15} \mathrm{sec}$. the neck is reduced further since atoms are ejected between two adjacent layers at the neck. In spite of the fact that the neck includes two atoms in $38<m<51$, the calculated conductance vary between $\sim 6 e^{2} / h$ and $2 e^{2} / h$. This demonstrates that $G(m)$ is strongly dependent on the atomic configuration when the neck becomes extremely thin (having one or two atoms) shortly before the break. In this situation, the overlap of the atomic potential from the well separated neighboring layers is weakened, but the potential variation due to the atoms at the neck becomes pronounced. This effect becomes pronounced by the reduced electron screening in very thin necks. On the other hand, owing to the finite range of atomic potentials, $G(m)$ becomes less sensitive to changes of atomic structure when the neck is thick and includes several atoms. As an example, $G(m)$ dipping in $39<m<44$ increases with increasing $m$ since the the atoms change from the $H$-site to the $T$-site registry as shown in Fig. 3. Such a dip was observed experimentally ${ }^{6}$. Note that such an effect is not taken into account neither by the nearly free electron $^{7,14}$ nor tight-binding calculations. ${ }^{13,35}$

The $G(m)$ curve calculated for the same wire by using $\Delta t=3 \times 10^{-15} \mathrm{sec}$ displays similar overall behaviors until $m=50$, except that jumps are delayed, the step structure becomes less apparent, and the fluctuations are pronounced. These are due to the fact that the atoms can be trapped in local minima when $\Delta t$ is small and hence structural changes can be retarded. Beyond $m>50$, the neck can survive for longer time since atoms cannot overcome the barrier in a short time. For $m>50, F_{z}(m)$ and hence $G(m)$ fluctuate; the cross section is reduced and weakened several times upon the atomic rearrangements, but it is recovered until the break. For the same reason, $G(m)$, slowly decreases in the region $65<m<75$ shortly before the break. Some of these conclusions are also derived from the recent conductance calculation that employs an orthogonalized nearest-neighbor $1 \mathrm{~s}$ tight-binding model with a half filled band and uses the atomic configurations revealed from the MD simulations of Au wire performed at $T=1 \mathrm{~K}$ by Todorov and Sutton. ${ }^{35}$

\section{MECHANICAL PROPERTIES OF NANOWIRES}

The effective force constant $k_{\text {eff }}$, which is defined as the mechanical force per elongation can be estimated from the slope of the curve $F_{z}(m)$. For a contact, the effective force constant was given by $k_{\mathrm{eff}}=R \gamma E /(1-\nu)^{2}$ (see Refs. 15 and 36). Here $R, \gamma, E$, and $\nu$ are, respectively, the contact radius, a factor describing the exact distribution of stress, Young's modulus and Poisson's ratio. From $F_{z}$ versus displacement curve measured by the combined STM and AFM, Agrait et al. ${ }^{15}$ obtained $k_{\text {eff }}=240 \mathrm{~N} / \mathrm{m}$ and $R \sim 3 \mathrm{~nm}$. Using the above contact expression of $k_{\text {eff }}$, they estimated $E \sim 66-44$ $\mathrm{GPa}$ which is consistent with the macroscopic value of the Young's modulus of Au. On the other hand, Stalder and Dürig ${ }^{16}$ found that the intrinsic yield strength $\left(\sigma_{Y}\right)$ of a neck with $R \sim 1 \mathrm{~nm}$ (corresponding to a cross-section $S \sim 300 \AA^{2}$ ) between the $\mathrm{Au}(111)$ sample and the Au tip is $8 \mathrm{GPa}$, which is one order of magnitude larger than the macroscopic value. The relatively larger value of intrinsic $\sigma_{Y}$ is attributed to the absence of dislocations in nanowires. However, the value of the intrinsic $\sigma_{Y}$ approached to $20 \mathrm{GPa}$ shortly before the break. This further increase of $\sigma_{Y}$ in the last step of the stretch, where the neck is reduced to $1-3$ atoms implies a structural change. In fact, as revealed from the present work the structure of the neck with a bundle of chains or single atomic chain is rather different from the original structure derived from the atomic arrangement of the macroscopic bulk and hence is expected to lead dramatic changes in the mechanical properties. For example, the observed "giant" yield strength may originate from the unusual changes in the structure. By definition, the yield strength $\sigma_{Y}$ is the stress, $\sigma=F_{z} / S$ at the yield point where the linear relation between stress and strain starts to break. Since $\sigma_{Y}=E \epsilon_{Y}\left(\epsilon_{Y}\right.$ being the strain at the yield point), the observed "giant" yield strength may be due to relatively higher strain value and/or higher Young's modulus attained by nanowires. In general, the high strain value is achieved in nanowires because the mechanism of yielding by dislocation is absent. Moreover, owing to the transition from the $H$-site registry to the $T$-site registry, a neck can attain relatively larger values ${ }^{26,27}$ for $\epsilon_{Y}$. On the other hand, $a b$ initio calculations that we discuss below demonstrate that $E$ also may increase in the neck consisting of atomic chain(s). This feature originates from the structural transformation induced by high strain in nanowires that leads to a change in bonding structure. Of course the yield strength and Young's modulus are definitions, which are developed 
within the continuum description of matter. By adopting some of the variables (such as the cross-section $S$ ) in these definitions we attempt to calculate $E$ in the quantum limit.

The Young's modulus of an atomic chain can be expressed by $E=k b / S$, where $k$ is the force constant and $b$ is the interatomic distance. Apparently, the size and dimensionality of the chain go beyond the limits of continuum description, and the estimation of $k$ requires $a b$ initio quantum mechanical calculations. To this end, we carry out total energy calculations by using SCF pseudopotential method in momentum representation. Since the $a b$ initio pseudopotential calculations for $\mathrm{Cu}$ chain involve certain difficulties owing to the $3 d$ states near the $4 s$, we therefore take into account the infinite $\mathrm{Al}$ chain whereby we can use periodic boundary condition and pseudopotentials which converge easily for a simple metal. For example, by using the kinetic energy cutoff $|\vec{k}+\vec{G}|<7.5 \mathrm{Ry}$, one can achieve accurate representation of the wave function and band energies. As pointed earlier, ${ }^{26,27}$ a short $\mathrm{Al}$ chain can form in an Al neck. Moreover, the mechanical properties of short Al chain can be revealed from those of infinite chain. We use nonlocal and norm-conserving pseudopotentials ${ }^{37}$ with the Wigner exchange-correlation potential. We treat the 1D infinite atomic chain in a 3D supercell geometry in which the interchain distance is taken $7.5 \AA$. The minimum total energy is obtained for $b=2.403 \AA$. The force constant $k$ is obtained from the variation of total energy relative to interatomic distance $b$, i.e., $k=-\partial^{2} E_{T}(b) / \partial b^{2}$, and is found $164 N / m$. There are ambiguities in the definition of cross section; here we used two schemes which yield similar values: (i) $S=0.38 \pi \lambda_{F}^{2}$ which is the cross section necessary to open a ballistic conduction channel within the free electron and infinite wall cylindrical potential approximation. (ii) $S=\pi R^{2}$; here $R$ is the radius at the value of calculated SCF potential, which is equal to $E_{F}$. For $\mathrm{Al}$, we find $S=15.38 \AA^{2}$ at $T=300 \mathrm{~K}$ from the first scheme, and $S \sim 14.0 \AA^{2}$ from the second scheme. Eventually, we calculate the Young's modulus of Al chain $E \sim 260 \mathrm{GPa}$ which is approximately four times larger than the bulk value. This implies that the mechanical properties of metal chain or bundle structure is different from those of bulk values, as well as from those of the neck or metal wires having relatively larger cross section, such as $S \sim 300 \AA^{2}$ as in the experiment. ${ }^{15}$ The raise of $E$ upon bundle or chain formation may have dominant contribution to the observed "giant" yield strength.

\section{DISCUSSION}

The present analysis show that the yielding and fracture mechanisms of a nanowire under uniaxial tensile force depend on its size, atomic structure, and temperature. There are, however, features which are common to all nanowires under uniaxial strain. The important aspects of the present study are summarized.

(1) We found that the deformation (or elongation) treated in this study generally occurs in two different and consecutive stages that repeat during the stretch. In the first stage, the stored strain energy and average tensile force increase with increasing stretch, while the layer structure persists. The fluctuations can occur possibly due to displacement and relocation of atoms within the same layer or atom exchange between adjacent layers. The variation of $F_{z}(m)$ in this stage is approximately linear, but it deviates from linearity as the number of atoms in the neck is reduced. This stage was identified as quasielastic. The second stage that follows each quasielastic stage is the yielding stage. A wire can yield by different mechanisms depending on its diameter. The motion of the dislocation and/or the slips on the glide planes are generally responsible for the yielding if the wire maintains an ordered (crystalline) structure and has a relatively larger cross section. The type of the ordered structure and its orientation relative to the axis are expected to affect the yielding. On the other hand, MD simulations predict different mechanical instabilities leading to yielding if the cross section of wires is small as considered in the present study. These are disorder-order transformation and single-atom process. Initially, once the elongation of the nanowire reaches the interlayer separation at the end of the quasielastic stage, the structure becomes disordered; but after a few increments of stretch, it is recovered with the formation of a new layer. In this yielding stage, $\left|F_{z}\right|$ decreases abruptly; the cross section of narrowest neck layers which determines the conductance, are reduced abruptly by few atoms. When the neck becomes very narrow (having 3-4 atoms) the yielding is realized, however, by single atom jumping from one of the adjacent layers to interlayer space. At low temperature, the yielding takes place in relatively shorter time interval within one or two stretch steps. Owing to the limited mobility of atoms at low temperature, the character of quasielastic stage changes if the neck is long enough. In this situation, each layer sequentially ejects one atom to the adjacent interlayer space as it widens with the applied strain. The layers at the neck are made from the pentagon or hexagon rings which become staggered in different layers. The off-layer atoms make a chain passing through the center of rings. In this new phase, the elastic and yielding stages are intermixed and elongation which is more than one interlayer distance can be accommodated. However, this structural instability result in slightly different structure if the time steps, $\Delta t$ is reduced in the MD simulations. In the initial stage of pulling off the single-atom process, in which individual atoms also migrate from central layers towards the end layers, can give rise to relatively smaller and also less discontinues changes in the cross section. The tendency to minimize the surface area and hence to reduce the energy of the system is the main driving force for this type of necking. Our simulation studies on the metal wires, that have an initial diameter of $\sim \lambda_{F}$ at the neck, but their diameters gradually increase as one goes away from the neck region, have indicated structural instabilities; those wires had further necking even in the absence of any tensile strain. This puzzling result is in comply with the recent experiment by using mechanical break junction on the gold neck that was broken by itself at room temperature. ${ }^{38}$

(2) In the initial steps of stretch, the layered structure and ordered 2D atomic arrangement within the layers are maintained. Upon increased uniaxial strain (increased $m$ ), the layers become wider and rougher due to the atoms departing from the atomic plane, the 2D lattice is distorted, and interatomic distances start to deviate from the bulk equilibrium value. In the elastic stage, one can still distinguish layer structure and some kind of order in the atomic arrangement within a layer. In particular, the H-site registry between 
layers are maintained if the layers contain enough number of atoms. The ordered atomic arrangement becomes disordered at low temperature towards the end of stretch.

(3) The quasielastic and following yielding stages are reminiscent of the stick-slip motion. In the course of stretch, elastic and yielding stages repeat; the surface of the nanowire roughens and deviates strongly from circular symmetry. The narrowest part of the neck, is usually only one layer thick, and is connected to the hornlike ends. In this circumstances the contribution of the tunneling becomes significant. Our results of atomic simulations point to the fact that neither adiabatic evolution of discrete electronic states, nor circular symmetry induced degeneracy can occur in the neck. Consequently any quantized sharp structure shall be smeared out by channel mixing and tunneling. ${ }^{11,12}$

(4) If the interlayer interaction is reduced as a result of extensive strain, the 2D atomic structure (squarelike lattice) is transformed into hexagonal rings. At prolonged stretch, just before the break, the cross section of layers at the central part of the neck is reduced to $2-3$ atoms. In this case, the $H$-site registry may change to the $T$-site registry. This leads to the formation of a bundle of atomic chains or of a single atomic chain. We consider this a dramatic change in the atomic structure of the wire and may have important implications.

(5) Our ab initio calculations of Young's modulus on the 1D metal chains indicate that the neck having chain structure may have yield strength much higher than the bulk value. This is in good agreement with experimental results, and also implies that the elastic properties of an atomic size neck deviate from those of the bulk defined in the continuum limit.

(6) Our calculations show that the conductance makes a sudden drop whenever a sudden atomic rearrangement in the neck [such as the formation of a new layer with relatively smaller cross section, the migration of atom(s) between two adjacent layers or the disordering that cause sudden decrease of the tensile force] occurs during the pulling off. Hence the
$G(s)$ curve is closely related with the structural changes during the stretch. In very thin necks including one or two atoms $G(s)$ becomes more sensitive to the atomic rearrangements. Recent studies demonstrated that not only the atomic structure, but also the bonding sites to the electrodes are crucial for the measured conductance. ${ }^{27}$

(7) The most important aspect of the nanowires that underlie various unusual (or novel) properties is that the spacings of the energy levels of transversally confined electronic states is wide and can easily be resolved. Even if the potential (or profile) of the wire is not uniform the level spacing is reflected to the related physical properties. In this respect the structure of the experimental $G(s)$ curve reflects the quantization of electronic states in the neck, but not the quantization of conductance.

(8) Nanowires under tensile force exhibits unusual structural properties. Further study of nanowires not only on their electron transport properties, but also on their electronic, magnetic, low temperature, mechanical, and energy transport properties are also expected to reveal that the effects of the wide level spacing can lead to discontinuous behavior in the heat conduction.

In conclusion, the simulations of atomic structure in nanowires under tensile stress indicate mechanisms of plastic deformation and elongation. When the restoring effect of the initial structure is weakened under the applied uniaxial force, the atomic arrangement deviates from the global minimum and can be stabilized in a different local minimum. The corresponding configuration exhibits a noncrystalline, shortrange order, and interesting mechanical and transport properties induced thereform.

\section{ACKNOWLEDGMENTS}

We would like to thank Professor M. S. Daw for providing the embedded atom potential for $\mathrm{Cu}$ and Professor Ş. Erkoç and Dr. Z. Güvenç for helpful discussions. In particular, we benefited from the discussion with Professor C. Y. Fong in the calculation of atomic pseudopotential.
${ }^{1}$ For extensive review see Condensed Systems of Low Dimensionality, NATO Advanced Study Institute, Series B253: Physics, edited by J. L. Beeby (Plenum Press, New York, 1991); Highlights in Condensed Matter Physics and Future Prospects, edited by Leo Esaki, NATO Advanced Study Institute, Series B285: Physics (Plenum Press, New York, 1991).

${ }^{2}$ J. K. Gimzewski, and R. Möller, Phys. Rev. B 36, 1284 (1987); J. K. Gimzewski, R. Möller, D. W. Pohl, and R. R. Schlittler, Surf. Sci. 189, 15 (1987); U. Dürig, J. K. Gimzewski, and D. W. Pohl, Phys. Rev. Lett. 57, 2403 (1986).

3 A. P. Sutton and J. B. Pethica, J. Phys. Condens. Matter 2, 5317 (1990).

${ }^{4}$ U. Landman, W. D. Luedtke, N. A. Burnham, and R. J. Colton, Science 248, 454 (1990); U. Landman and W. D. Luedtke, J. Vac. Sci. Technol. B 9, 414 (1991).

${ }^{5}$ N. Agraï, J. G. Rodrigo, and S. Vieria, Phys. Rev. B 47, 12345 (1993)

${ }^{6}$ J. I. Pascual, J. Méndez, J. Gómez-Herrero, A. M. Baró, N. Garcia, and V. T. Binh, Phys. Rev. Lett. 71, 1852 (1993); J. I.
Pascual, J. I. Méndez, J. Gómez-Herrero, A. M. Baró, N. Garcia, U. Landman, W. D. Luedtke, E. N. Bogachek, and H. P. Cheng, Science 267, 1793 (1995).

${ }^{7}$ L. Olesen, E. Laegsgaard, I. Stensgaard, F. Besenbacher, J. Schiøtz, P. Stoltze, K. W. Jacobsen, and J. K. Norskov, Phys. Rev. Lett. 72, 2251 (1994); 74, 2147 (1995).

${ }^{8}$ J. M. Krans, C. J. Muller, I. K. Yanson, T. C. M. Govaert, R. Hesper, and J. M. Van Ruitenbeek, Phys. Rev. B 48, 14721 (1994); J. M. Krans, C. J. Muller, N. Van der Post, F. R. Postma, A. P. Sutton, T. N. Todorov, and J. M. Van Ruitenbeek, Phys. Rev. Lett. 74, 2146 (1995).

${ }^{9}$ N. D. Lang, Phys. Rev. B 36, 8173 (1987).

${ }^{10} \mathrm{~J}$. Ferrer, A. Martin-Rodero, and F. Flores, Phys. Rev. B 38, 10113 (1988).

${ }^{11}$ S. Ciraci and E. Tekman, Phys. Rev. B 40, 11969 (1989); S. Ciraci, Tip-surface Interactions in Scanning Tunneling Microscopy and Related Methods, edited by R. J. Bohm, N. Garcia, and H. Rohrer (Kluwer, New York, 1990), Vol. 184, p. 113.

${ }^{12}$ E. Tekman and S. Ciraci, Phys. Rev. B 43, 7145 (1991). 
${ }^{13}$ T. N. Todorov and A. P. Sutton, Phys. Rev. Lett. 70, 2138 (1993).

${ }^{14}$ A. M. Bratkovsky, A. P. Sutton, and T. N. Todorov, Phys. Rev. B 52, 5036 (1995).

${ }^{15}$ N. Agraï, G. Rubio, and S. Vieira, Phys. Rev. Lett. 74, 3995 (1995); G. Rubio, N. Agraï, and S. Vieira, ibid. 76, 2302 (1996).

${ }^{16}$ A. Stalder and U. Dürig, Appl. Phys. Lett. 68, 637 (1996).

${ }^{17}$ S. Ciraci and I. P. Batra, Phys. Rev. B 33, 4294 (1986); I. P. Batra, S. Ciraci, G. P. Srivastava, J. S. Nelson, and C. Y. Fong, ibid. 34, 8246 (1986).

${ }^{18}$ R. M. Lynden-bell, Science 263, 1704 (1994).

${ }^{19}$ M. S. Daw and M. I. Baskes, Phys. Rev. B 29, 6443 (1984).

${ }^{20}$ S. M. Foiles, M. I. Baskes, and M. S. Daw, Phys. Rev. B 33, 7983 (1986).

${ }^{21}$ M. S. Daw, Phys. Rev. B 39, 7441 (1989).

${ }^{22}$ A. P. Sutton, Curr. Opinion Solid State Mater. Sci. 1, 827 (1996).

${ }^{23}$ The stretching velocity $v_{s}$ defined in the text is taken a measure of the strain rate even if it is not directly comparable with the strain rate in experiment. $\Delta t=10^{-13} \mathrm{sec}$ is an extreme limit in the MD simulations. It is most interesting that the wires have been found stable when they are relaxed with $\Delta t=10^{-13} \mathrm{sec}$. Deviation from the simulations that used relatively smaller time steps $\Delta t=1.5 \times 10^{-14} \mathrm{sec}$ and $3 \times 10^{-15} \mathrm{sec}$ are found to be negligible when the cross section of the wire is large. The large time step was also convenient to bring the atom to the global minimum.

${ }^{24}$ The increase of $F_{z}(m)$ between two consecutive jumps may deviate from the linear behavior for the reason outlined in Sec. VI. The elastic and yielding stages were predicted earlier in Refs. 3, 4,15 , and 16 .
${ }^{25}$ Note that the layers are not planar and they have finite thickness owing to the motion of atoms. We specify the layer structure if all its atoms are separated from those of the adjacent layers by a significant distance.

${ }^{26}$ See, for example, S. Ciraci, A. Baratoff, and I. P. Batra, Phys. Rev. B 42, 7618 (1990) for Al(111) surface; and S. Ciraci, E. Tekman, A. Baratoff, and I. P. Batra, ibid. 46, 10411 (1992) for $\mathrm{Al}(001)$ surface.

${ }^{27}$ H. Mehrez, S. Ciraci, A. Buldum, and I. P. Batra, Phys. Rev. B 55, R1981 (1997).

${ }^{28}$ In the hexagonal structure, the arrangements of the structure are reminiscent 2D hexagonal lattice, but angles are distorted, first nearest-neighbor distances are changed and consequently the six-fold rotation symmetry is broken.

${ }^{29}$ Yu. V. Sharvin, Zh. Éksp. Teor. Fiz. 48, 984 (1965) [Sov. Phys. JETP 21, 655 (1965)].

${ }^{30}$ E. Tekman and S. Ciraci, Phys. Rev. B 40, 8559 (1989).

${ }^{31}$ I. Kander, Y. Imry, and U. Sivan, Phys. Rev. B 41, 12941 (1990).

${ }^{32}$ H. Mehrez, M.S. thesis, Bilkent University, 1996.

${ }^{33}$ D. R. Hamann, Phys. Rev. B 40, 2980 (1989).

${ }^{34}$ D. M. Ceperley and B. J. Alder, Phys. Rev. Lett. 45, 566 (1980).

${ }^{35}$ T. N. Todorov and A. P. Sutton, Phys. Rev. B 54, R14 234 (1996).

${ }^{36}$ K. L. Johnson, Contact Mechanics (Cambridge University Press, Cambridge, 1986).

${ }^{37}$ G. B. Bachelet, D. R. Hamann, and M. Schlüter, Phys. Rev. B 26, 4199 (1982).

${ }^{38}$ C. J. Muller, J. M. Krans, T. N. Todorov, and M. A. Reed, Phys. Rev. B 53, 1022 (1996). 